\title{
USO PROBLEMÁTICO DE INTERNET Y CALIDAD DE VIDA EN UNIVERSITARIOS DE UNA INSTITUCIÓN PRIVADA CONFESIONAL DE Medellín, Colombia, 2017
}

\author{
Germán Arturo González Anaya ${ }^{1}$ \\ Facultad de Ingeniría, Corporación Universitaria Adventista, \\ Colombia
}

Recibido: 20 de mayo de 2017 Aceptado: 17 de junio de 2017

\begin{abstract}
Resumen
El objeto de este estudio es observar la relación entre el uso problemático del internet y la calidad de vida en estudiantes universitarios. Se llevó a cabo por medio de un estudio no experimental con un enfoque descriptivo con una muestra de 302 universitarios entre 16 y 25 años, siendo 51.3\% varones y 48.7\% mujeres utilizando el Internet Adicción Test (IAT) (1998) de Young adaptado al español y el Índice Multicultural de Calidad de Vida (MQLI) (2000) de Mezzich, Cohen, Ruiperez, Banzato, y Zapata-Vega, los dos instrumentos validados en Colombia. Los resultados identificaron un 9.9\% de los participantes con uso problemático de internet y un 49\% con problemas frecuentes por el uso del mismo. El 49\% de los estudiantes utiliza internet cinco horas o más al día. Se encontró una correlación negativa y altamente significativa entre el uso problemático del internet y la calidad de vida (rho= -.371, p<.01), expresado en una correlación significativa y negativa con la satisfacción con la integridad personal (rho= -.359, $\mathrm{p}<.01)$, la satisfacción con el funcionamiento productivo ( $r h o=-.280$, $\mathrm{p}<.01)$, la satisfacción social ( $r h o=-.159, \mathrm{p}<.01$ ) y la satisfacción con el sentido de vida (rho= -.296, p<.01). Fue posible concluir que, al aumentar el uso problemático del internet en los universitarios, disminuirá el nivel de su calidad de vida.
\end{abstract}

Palabras Clave: uso problemático del Internet, calidad de vida, adicción a internet

\begin{abstract}
The aim of this study is to observe the relationship between the problematic use of the Internet and the quality of life in university students. It was a non-experimental study with a descriptive approach. The sample consisted of 302 university students between 16 and 25 years old, 51.3\% male and 48.7\% female. The instrument used was the Internet Addiction Test (IAT) (1998) of Young adapted to spanish and the Multicultural Quality of Life Index (MQLI) (2000) of Mezzich, Cohen, Ruiperez, Banzato, and Zapata-Vega, the two instruments validated in Colombia. The results identified 9.9\% of the participants with problematic internet use and 49\% with frequent problems due to its use. It was possible to observe 49\% of students use the internet for five hours or more a day. A negative and highly significant correlation was found between problematic internet use and quality of life (rho = -371 , $\mathrm{p}<.01)$. The dimensions of quality of life that also had a significant and negative correlation with the problematic use of the Internet were: satisfaction with personal integrity (rho $=-.359, \mathrm{p}<.01$ ), satisfaction with productive functioning ( rho $=-.280, \mathrm{p}<.01)$, social satisfaction
\end{abstract}


Revista de Investigación Universitaria | Volumen 6 - Número 2, Julio - Diciembre, 2017 | ISSN 2078-4015 (En línea) DOI :

(rho $=-.159, \mathrm{p}<.01)$ and satisfaction with the meaning of life (rho = -.296, $\mathrm{p}<.01)$. It was possible to conclude that, by increasing the problematic use of the internet in university students, the level of their quality of life will decrease.

Keywords: problematic use of internet, quality of life, internet addiction 


\section{Introducción}

Entre el año 2000 y el 2017 el acceso global a internet ha aumentado un 936\% llegando a un índice de penetración del 49.7\% de la población mundial (Miniwatts Marketing Group, 2017). En Latinoamerica, en una medición reciente realizada en Brasil, México, Argentina, Colombia, Perú y Chile ese índice alcanza el 56,1\% (IMS y comscore, 2016). A nivel de Colombia el informe señala que el 57,8\% de los habitantes tienen acceso a la red.

El uso tan generalizado de internet tiene el potencial de afectar de una manera u otra la calidad de vida de los jóvenes, el presidente de la Asociación Psiquiátrica de América Latina Alfredo Cía afirma que "la utilización de internet con fines recreativos, excesiva y persistente puede conducir a una adicción. De hecho, su importancia clínica y epidemiológica la sitúa como una de las patologías emergentes de mayor impacto en el siglo actual" (Cía, 2014, p.214).

Una de las denominación adoptada para la adicción a internet es el "Uso Problemático de Internet" (UPI) que se puede definir como la incapacidad del individuo para controlar el uso del internet lo cual causa malestar a nivel psicológico y afecta su funcionamiento (Gracia, Vigo, Fernández y Marcó, 2002).

En Colombia Puerta-Cortés y Carbonell (2013) encontraron que el 52\% de los participantes de su estudio del uso de internet y su relación con la salud, se encontraba en un estado de adicción leve a internet y un 1,9\% en un estado de adicción severa, los hallazgos de Frangos y Kiohos (2010) y Dalbudak y Evren (2014) revelan porcentajes de 34,2\% y $58,6 \%$ respectivamente de estudiantes con algún grado de adicción a internet. Por otra parte, algunos estudios (Hilt, Bouvet de Korniejezuk, y Collins, 2015; Şenormancla etal., 2014) muestran índices muy bajos de dicha adicción en los participantes. 
Por otra parte cómo un concepto vital para desarrollo del ser humano, se ha hecho cada vez más conocido el término "calidad de vida", esta se puede definir como "un grado óptimo de la satisfacción de las necesidades humanas" (Alguacil, 1998, p.73).

Grimaldo (2012) realizó en el Perú su estudio Calidad de vida en estudiantes de secundaria de la ciudad de Lima, con 589 estudiantes de cuarto y quinto año de secundaria de centros educativos estatales, utilizando la Escala de Calidad de Vida de Olson y Barnes, su investigación halló un promedio de calidad de vida óptimo en hombres y mujeres del nivel socio económico medio y bajo, la autora del estudio señala que la percepción subjetiva de los participantes, antes que los indicadores objetivos, hace que los mismos tengan una visión positiva de su situación. En dicho estudio no se halló relación entre la Calidad de Vida CV con el UPI.

Sin embargo Mirzazadeh et al., (2016) establecieron que la calidad de vida se veía afectada negativamente por la adicción a internet en relación al dominio físico, social y psicológico. También se encontró que los estudiantes en tal condición tenían menor rendimiento académico.

Esta relación entre CV y UPI realza su importancia al considerar la integralidad del ser humano como un principio inalterable, que implica un sujeto integral y por lo tanto un desarrollo integral y equilibrado del mismo, que abarca todo el ser y toda la experiencia vital del hombre e incluye las facultades físicas, mentales y espirituales (White, 2009).

El UPI Es la incapacidad para controlar el uso de Internet, que eventualmente involucra aspectos psicológicos, sociales, problemas académicos y / o profesionales en la vida de una persona (Young, 1997). El criterio diagnóstico de Young señala que se puede establecer la adicción a internet cuándo el usuario responde a cinco o más de las siguientes preguntas 
durante un período de 6 meses, esto permite diferenciar y establecer el uso compulsivo de internet de su uso normal.

1. Sentimiento de preocupación por Internet (piensa acerca de anteriores o futuras actividades en línea).

2. Siente la necesidad de aumentar la cantidad de tiempo usando Internet para alcanzar satisfacción.

3. Ha realizado esfuerzos repetidos sin éxito para controlar, disminuir o detener el uso de Internet.

4. Se ha sentido inquieto, malhumorado, deprimido o irritable en sus intentos de parar o detener el uso de Internet.

5. Ha estado más tiempo del que pretendía en Internet.

6. Ha estado en riesgo de perder alguna relación importante, un trabajo o una oportunidad de educación debido a Internet.

7. Ha mentido a miembros de su familia, al terapeuta o a otros para ocultar la importante relación con Internet.

8. Usa Internet como camino para escapar de problemas o para aliviar un disgusto (sentimientos de impotencia, ansiedad, depresión o culpa).

Puerta-Cortés, Carbonell, y Chamarro, (2013) han podido establecer a través del análisis factorial tres dimensiones principales que son sustentadas en la propuesta de Young como se puede observar en la figura 1.

Figura 1 Dimensiones de la adicción a internet

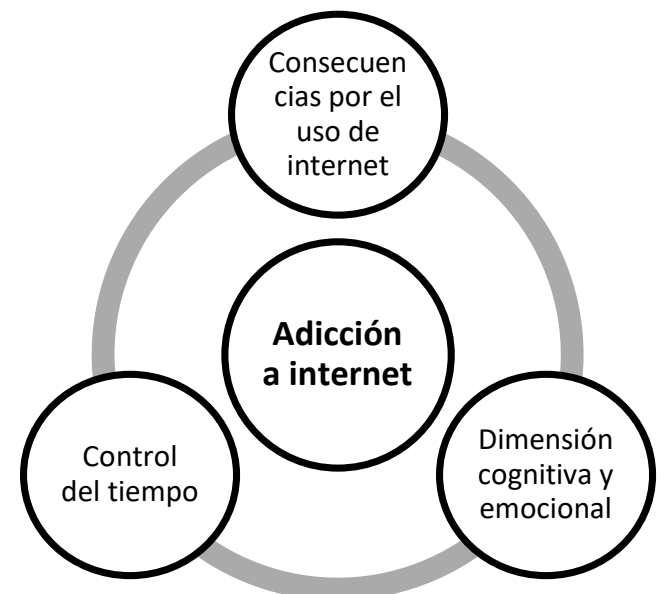

Fuente: Elaboración propia

Cómo un enfoque diferente a la tendencia actual de clasificar al UPI como una adicción conductual, se halla la propuesta de Van Roij y Prause, (2014) quienes se presentan a favor de abandonar los comportamientos 
Revista de Investigación Universitaria | Volumen 6 - Número 2, Julio - Diciembre, 2017 | ISSN 2078-4015 (En línea)

DOI :

(cc) BY

relacionados con internet con las etiquetas de problemáticos, excesivos, adicción, dependencia, patológico, impulsivo, compulsivo, anormal o con los prefijo "hiper" que señalan un estado de enfermedad. Proponen el término "alta frecuencia en el uso de internet" y la posibilidad de abordar la frecuencia e intensidad de los comportamientos empoderando al individuo para generar cambios.

La felicidad del ser humano está estrechamente con su calidad de vida y esta es lograda con la satisfacción de las necesidades de la persona sin importar su raza. (Mcalls citado por Salas y Garzón, 2013), por esta razón es de alto valor el esfuerzo para comprender e investigar la calidad de vida, pues ese empeño en ser feliz es propio de cada persona en el planeta. Junto con ese deseo, la búsqueda de la vida larga y buena a lo largo de la existencia en el planeta es algo a lo cual los seres humanos le dan gran valor y que anhelan con intensidad, de manera que tratan de obtener el desarrollo de sus capacidades que les brinda finalmente un sentimiento igualmente buscado, la libertad (Sen, 1999).

La reflexión y el análisis de la calidad de vida ayuda no solamente a observar la satisfacción, la felicidad, el bienestar subjetivo y la abundancia, pues también permite contrastar el dolor, la enfermedad, las limitaciones y las necesidades no satisfechas de las personas y los pueblos, lo cual es de gran valor para la búsqueda de soluciones en el esfuerzo porque esa calidad de vida mejore (Moreno y Ximénez, 1996).

En relación a la CV Ardila (2003) la define de la siguiente manera:

La calidad de vida es un estado de satisfacción general, derivado de la realización de las potencialidades de la persona. Posee aspectos subjetivos y aspectos objetivos. Es una sensación 
subjetiva de bienestar físico, psicológico y social. Incluye como aspectos subjetivos la intimidad, la expresión emocional, la seguridad percibida, la productividad personal y la salud objetiva. Como aspectos objetivos el bienestar material, las relaciones armónicas con el ambiente físico y social y con la comunidad, y la salud objetivamente percibida (p.164)

Se ha desarrollado el concepto de Calidad de Vida con diversos enfoques como ha sido observado por Felce y Perry (Espinosa, 2014) y se resume en la figura 2.

Figura 2 Concepto calidad de vida con cuatro enfoques

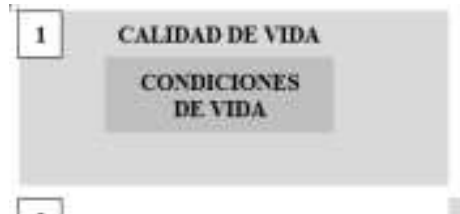

\section{SAnsFiccióx}

PrRSONAL

2

CONDICIONES DE VIDA

CALIDAD DE VIDA

sainsfacelox PEISONAL

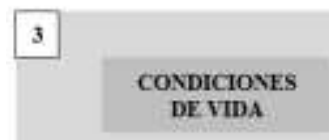

CAIIDAD DE VWA DE VIDA

\section{SATISACCLó} PrRsoyt.

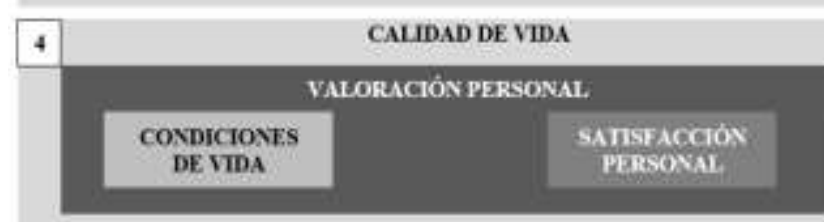

Fuente: Aproximación teórica al concepto de calidad de vida (Espinosa, 2014, p.339).

Estos modelos han definido líneas de discusión que afectan el desarrollo de esta área de estudio. 
Los modelos han sido planteados la CV teniendo en cuenta la CV en sus factores objetivos, subjetivos o ambos añadidos a los valores.

\section{Metodología}

\subsection{Diseño y tipo de investigación}

El presente estudio corresponde a un enfoque cuantitativo de tipo descriptivo, transversal y con diseño no experimental.

\subsection{Participantes}

Para la realización de este estudio se utilizó un muestreo no probabilístico por conveniencia, los participantes fueron 302 estudiantes de pregrado de entre los 16 y 25 años de la Corporación Universitaria Adventista, en Medelín Colombia. El 51.3\% fueron hombres y el 48.7\% mujeres. La tabla 1 presenta la clasificación en función al género, el rango de edad, el lugar de residencia y su ocupación.

Tabla 1

Características de la muestra en estudio

\begin{tabular}{lcl}
\hline & $N$ & $\frac{\circ}{0}$ \\
\hline Sexo & 153 & $51.3 \%$ \\
Masculino & 145 & $48.7 \%$ \\
Femenino & & \\
Edad & 137 & $45.4 \%$ \\
16 a 20 años & 165 & $54.6 \%$ \\
21 a 25 años & & $33.6 \%$ \\
Residencia & 99 & $38.6 \%$ \\
Residencia & & \\
universitaria & 114 & $27.8 \%$ \\
Reside con la & & \\
familia & 82 & \\
Habitación en & & \\
arriendo & 130 & $55.9 \%$ \\
Ocupación & 165 & \\
Solo estudia & & \\
Estudia y trabaja & & \\
\hline
\end{tabular}

\subsection{Instrumentos}

Los instrumentos aplicados fueron el Test de Adicción a Internet (Internet Addiction Test; IAT, por su siglas en 
inglés) desarrollado por Kimberly Young en 1996, el cual ha sido adaptado a diferentes idiomas (Alavi et al., 2010 ; Barke, Nele, y Kröner-Herwig, 2012; Chang, Law, y Pui, 2007; Chong, Saramah, Aili, Subash, y Manveen, 2012; Khazaal et al., 2008). El IAT es un instrumento para la evaluación del uso de internet a fin de percibir problemas o adicción. Está conformado por 20 ítems con preguntas cerradas. Las dimensiones que conforman el instrumento son:

1. Consecuencias de uso de internet: $6,7,8,5,2,4,14$

2. Dimensión cognitiva-emocional: 15, 20, 18, 19, 10, 9, $13,11,3,12$

3. 3. Control del tiempo: 1,16,17

La versión utilizada en este estudio es la versión en idioma español se registró el coeficiente Alfa de Cronbach de 0.882. El instrumento utiliza una escala Likert de cinco puntos ( 1 = rara vez, a 5 = siempre). El puntaje mínimo posible es 20 y el máximo 100. Los puntajes de 20 a 49 tienen correspondencia con un uso controlado de Internet, de 50 a 79 indica problemas frecuentes por el uso de Internet y un puntaje mayor a 80 se refiere a problemas significativos en la vida debido al uso de Internet.

La segunda escala utilizada fue el Índice Multicultural de Calidad de Vida (MQLI) Mezzich, Cohen, Ruiperez, Banzato, y Zapata-Vega (2011), ha sido validado en cuatro idiomas: español, inglés, chino y coreano. La versión en español (MQLI-Sp) ha sido utilizada en otros países, como Venezuela España, Chile y Perú (Jatuff, Zapata-Vega, Montenegro, y Mezzich, 2007). Sus dimensiones son:

1. Satisfacción con la integridad personal: Ítems 1,2,3

2. Satisfacción con el funcionamiento productivo: Ítems $4,5,6$

3. Satisfacción social: Ítem7

4. Satisfacción con el sentido vital: Ítems 8,9,10

Tiene diez ítems relacionados con bienestar físico, bienestar psicológico, autocuidado y funcionamiento 
independiente, funcionamiento ocupacional, funcionamiento interpersonal, apoyo social-emocional, apoyo comunitario y de servicios, plenitud personal, satisfacción espiritual y calidad de vida global. Utiliza una escala ordinal de diferencial semántico de 1 a 10 puntos, donde 1 es malo y 10 es excelente. Se halló una consistencia interna del instrumento con coeficiente del Alfa de Cronbach de 0.863.

\subsection{Análisis de datos}

El procesamiento de los datos se realizó por medio del programa estadístico SPSS, versión 23. Se llevaron a cabo análisis de frecuencia para identificar las variables sociodemográficas, la relación entre uso problemático de internet según la edad y el sexo. Asimismo, el índice de calidad de vida con la edad y sexo de los participantes. Luego de evaluar la distribución normal de las variables por medio de Kolmogorov-Smirnow (K-S), se utilizó el rho de Spearman para el análisis de correlación entre el uso problemático de internet y la calidad de vida.

\section{Resultados}

\subsection{Resultados}

\subsubsection{Resultados 1}

Se aprecia en la tabla 2 que el 49\% de los estudiantes presentan problemas frecuentes relacionados al uso del internet y el 9.9\% presentan problemas significativos. Con respecto a los componentes de la adicción a internet, se observa que el 13.9\% presentan consecuencias graves en su vida que incluyen malestar del entorno social cercano, disminución del rendimiento académico, descuido de las actividades de la casa y no dormir toda la noche por estar conectado a internet. También se aprecia que el 48.7\% de los estudiantes presentan problemas frecuentes en el área cognitivo y emocional y la mayoría presenta problemas frecuentes para controlar el tiempo que pasa en internet $(52.6 \%)$. 
Tabla 2

Nivel de uso problemático de internet

\begin{tabular}{|c|c|c|c|c|c|c|}
\hline & \multicolumn{2}{|c|}{ Control } & \multicolumn{2}{|c|}{$\begin{array}{l}\text { Problemas } \\
\text { frecuentes }\end{array}$} & \multicolumn{2}{|c|}{$\begin{array}{l}\text { Problemas } \\
\text { significativos }\end{array}$} \\
\hline & $\mathrm{n}$ & $\frac{\circ}{0}$ & $\mathrm{n}$ & $\frac{\circ}{0}$ & $\mathrm{n}$ & $\frac{\circ}{\circ}$ \\
\hline Adicción a internet & 124 & 41.1 & 148 & 49.0 & 30 & 9.9 \\
\hline $\begin{array}{l}\text { Consecuencias de la } \\
\text { adicción al internet }\end{array}$ & 131 & 43.4 & 129 & 42.7 & 42 & 13.9 \\
\hline $\begin{array}{l}\text { Factores cognitivos } \\
\text { y emocionales }\end{array}$ & 127 & 42.1 & 147 & 48.7 & 28 & 9.3 \\
\hline Control del tiempo & 127 & 42.1 & 159 & 52.6 & 16 & 5.3 \\
\hline
\end{tabular}

\subsubsection{Resultados 2}

Se encontró que el $11.8 \%$ de los varones presentan problemas significativos relacionados al uso del internet y el 8.3\% de las mujeres también presentan dicho problema. Además, se observó que más de la mitad de los varones y mujeres presentan indicadores de adicción a internet. Ver tabla 3.

Tabla 3

Presencia de uso problemático de internet según sexo

\begin{tabular}{|c|c|c|c|c|c|c|}
\hline & \multicolumn{2}{|c|}{ Control } & \multicolumn{2}{|c|}{$\begin{array}{l}\text { Problemas } \\
\text { frecuentes }\end{array}$} & \multicolumn{2}{|c|}{$\begin{array}{l}\text { Problemas } \\
\text { significativos }\end{array}$} \\
\hline & $\mathrm{N}$ & $\frac{\circ}{0}$ & $\mathrm{n}$ & $\frac{\circ}{0}$ & $\mathrm{n}$ & $\frac{\circ}{0}$ \\
\hline \multicolumn{7}{|l|}{ Masculinoa } \\
\hline Adicción a internet & 63 & 41.2 & 72 & 47.1 & 18 & 11.8 \\
\hline $\begin{array}{l}\text { Consecuencias de la } \\
\text { adicción al internet }\end{array}$ & 71 & 46.4 & 59 & 38.6 & 23 & 15.0 \\
\hline $\begin{array}{l}\text { Factores cognitivos y } \\
\text { emocionales }\end{array}$ & 62 & 40.5 & 75 & 49.0 & 16 & 10.5 \\
\hline $\begin{array}{l}\text { Control del tiempo } \\
\text { Femeninob }\end{array}$ & 69 & 45.1 & 78 & 51.0 & 6 & 3.9 \\
\hline Adicción a internet & 59 & 40.7 & 74 & 51.0 & 12 & 8.3 \\
\hline $\begin{array}{l}\text { Consecuencias de la } \\
\text { adicción al internet }\end{array}$ & 56 & 38.6 & 70 & 48.3 & 19 & 13.1 \\
\hline $\begin{array}{l}\text { Factores cognitivos y } \\
\text { emocionales }\end{array}$ & 63 & 43.4 & 70 & 48.3 & 12 & 8.3 \\
\hline Control del tiempo & 55 & 37.9 & 80 & 55.2 & 10 & 6.9 \\
\hline
\end{tabular}

En la tabla 4 es posible identificar que el 13.1\% de los participantes de 16 a 20 años presentan problemas significativos de adicción a internet a diferencia del grupo de 21 a 30 años (7.3\%). De manera similar el 15.3\% de los más jóvenes (16 a 20 años) presentan problemas significativos 
como malestar del entorno social cercano, disminución del rendimiento académico, descuido de las actividades de la casa y no dormir toda la noche por estar conectado a internet. En cuanto a los factores cognitivos y emocionales se aprecia que hay más jóvenes (16 a 20 años) que presentan problemas significativos (12.4\%) que las personas mayores de 20 años $(6.7 \%)$.

Tabla 4

Presencia de uso problemático de internet según edad

\begin{tabular}{|c|c|c|c|c|c|c|}
\hline & \multicolumn{2}{|c|}{ Control } & \multicolumn{2}{|c|}{$\begin{array}{l}\text { Problemas } \\
\text { frecuentes }\end{array}$} & \multicolumn{2}{|c|}{$\begin{array}{l}\text { Problemas } \\
\text { significativos }\end{array}$} \\
\hline & $\mathrm{N}$ & $\frac{\circ}{0}$ & $\mathrm{~N}$ & $\frac{\circ}{0}$ & $\mathrm{n}$ & $\frac{\circ}{\circ}$ \\
\hline 16 a 20 años ${ }^{a}$ & & & & & & \\
\hline Adicción a internet & 43 & 31.4 & 76 & 55.5 & 18 & 13.1 \\
\hline $\begin{array}{l}\text { Consecuencias de la } \\
\text { adicción al internet }\end{array}$ & 49 & 35.8 & 67 & 48.9 & 21 & 15.3 \\
\hline $\begin{array}{l}\text { Factores cognitivos y } \\
\text { emocionales }\end{array}$ & 48 & 35.0 & 72 & 52.6 & 17 & 12.4 \\
\hline $\begin{array}{l}\text { Control del tiempo } \\
21 \text { a } 30 \text { años }\end{array}$ & 46 & 33.6 & 82 & 59.9 & 9 & 6.6 \\
\hline Adicción a internet & 81 & 49.1 & 72 & 43.6 & 12 & 7.3 \\
\hline $\begin{array}{l}\text { Consecuencias de la } \\
\text { adicción al internet }\end{array}$ & 82 & 49.7 & 62 & 37.6 & 21 & 12.7 \\
\hline $\begin{array}{l}\text { Factores cognitivos y } \\
\text { emocionales }\end{array}$ & 79 & 47.9 & 75 & 45.5 & 11 & 6.7 \\
\hline Control del tiempo & 81 & 49.1 & 77 & 46.7 & 7 & 4.2 \\
\hline
\end{tabular}

\subsubsection{Resultados 3}

Un el 28.8\% de los estudiantes evaluados presentan un nivel alto de calidad de vida, y la gran parte de los estudiantes presentan un nivel moderado de calidad de vida. Con respecto a sus componentes solo el $25.5 \%$ presentan un nivel alto de satisfacción con su integridad personal. Cifras inferiores se aprecian en los demás componentes de la calidad de vida siendo la satisfacción personal la más baja de ellas $(16,9 \%)$. Tabla 5 .

Tabla 5 


\section{$(\mathrm{cc}) \mathrm{EY}$}

Indice de calidad de vida

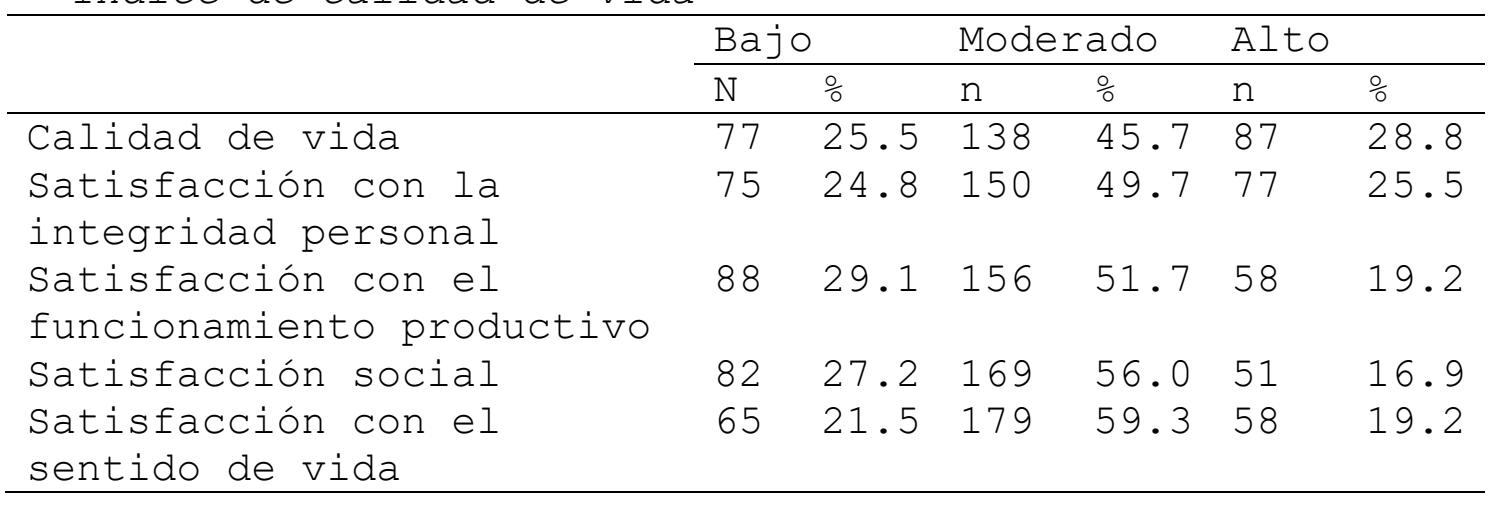

\subsubsection{Resultados 4}

En la tabla 6 se observa que casi la mitad de varones $(47.7 \%)$ y mujeres (44.1\%) presentan un nivel moderado de calidad de vida. Con respecto a sus componentes el 32\% de los varones presentan un nivel alto de satisfacción con su integridad personal a diferencia de las mujeres (17.9\%). Con respecto a los demás componentes la mayoría de los estudiantes presentan un nivel moderado de satisfacción con el funcionamiento productivo, satisfacción personal y satisfacción con el sentido de vida.

Tabla 6

Índice de calidad de vida según sexo

\begin{tabular}{|c|c|c|c|c|c|c|}
\hline & \multicolumn{2}{|c|}{ Bajo } & \multicolumn{2}{|c|}{ Moderado } & \multicolumn{2}{|c|}{ Alto } \\
\hline & $\mathrm{N}$ & $\frac{\circ}{0}$ & $\mathrm{n}$ & $\frac{\circ}{0}$ & $\mathrm{n}$ & $\frac{\circ}{0}$ \\
\hline \multicolumn{7}{|l|}{ Masculinoa } \\
\hline Calidad de vida global & 36 & 23.5 & 73 & 47.7 & 44 & 28.8 \\
\hline Satisfacción con la & 31 & 20.3 & 73 & 47.7 & 49 & 32.0 \\
\hline integridad personal & & & & & & \\
\hline $\begin{array}{l}\text { Satisfacción con el } \\
\text { funcionamiento productivo }\end{array}$ & 44 & 28.8 & 83 & 54.2 & 26 & 17.0 \\
\hline Satisfacción social & 46 & 30.1 & 89 & 58.2 & 18 & 11.8 \\
\hline $\begin{array}{l}\text { Satisfacción con el } \\
\text { sentido de vida } \\
\text { Femeninob }\end{array}$ & 32 & 20.9 & 92 & 60.1 & 29 & 19.0 \\
\hline Calidad de vida global & 40 & 27.6 & 64 & 44.1 & 41 & 28.3 \\
\hline $\begin{array}{l}\text { Satisfacción con la } \\
\text { integridad personal }\end{array}$ & 43 & 29.7 & 76 & 52.4 & 26 & 17.9 \\
\hline $\begin{array}{l}\text { Satisfacción con el } \\
\text { funcionamiento productivo }\end{array}$ & 43 & 29.7 & 71 & 49.0 & 31 & 21.4 \\
\hline Satisfacción social & 35 & 24.1 & 78 & 53.8 & 32 & 22.1 \\
\hline $\begin{array}{l}\text { Satisfacción con el } \\
\text { sentido de vida }\end{array}$ & 32 & 22.1 & 85 & 58.6 & 28 & 19.3 \\
\hline
\end{tabular}


En relación a los grupos etarios, se aprecia en la tabla 7 que el 33.9\% de los estudiantes de 21 a 25 años presentan un nivel alto de calidad de vida a diferencia de los estudiantes de 16 a 20 años, donde solo el 22.6\% tiene un nivel alto de satisfacción. Con respecto a este último grupo la mayoría presenta un nivel moderado de satisfacción en las diferentes áreas de la calidad de vida.

Tabla 7

Indice de calidad de vida según edad

\begin{tabular}{|c|c|c|c|c|c|c|}
\hline & \multicolumn{2}{|c|}{ Bajo } & \multicolumn{2}{|c|}{ Moderado } & \multicolumn{2}{|c|}{ Alto } \\
\hline & $\mathrm{N}$ & $\%$ & $n$ & $\frac{0}{0}$ & $\mathrm{n}$ & $\frac{\circ}{0}$ \\
\hline 16 a 20 años $^{a}$ & & & & & & \\
\hline Calidad de vida global & 34 & 24.8 & 72 & 52.6 & 31 & 22.6 \\
\hline $\begin{array}{l}\text { Satisfacción con la } \\
\text { integridad personal }\end{array}$ & 35 & 26.3 & 69 & 50.4 & 32 & 23.4 \\
\hline $\begin{array}{l}\text { Satisfacción con el } \\
\text { funcionamiento productivo }\end{array}$ & 43 & 31.4 & 69 & 50.4 & 25 & 18.2 \\
\hline Satisfacción social & 38 & 27.7 & 76 & 55.5 & 23 & 16.8 \\
\hline $\begin{array}{l}\text { Satisfacción con el } \\
\text { sentido de vida } \\
21 \text { a } 25 \text { añosb }\end{array}$ & 34 & 24.8 & 83 & 60.6 & 20 & 14.6 \\
\hline Calidad de vida global & 43 & 26.1 & 66 & 40.0 & 56 & 33.9 \\
\hline $\begin{array}{l}\text { Satisfacción con la } \\
\text { integridad personal }\end{array}$ & 39 & 23.6 & 81 & 49.1 & 45 & 27.3 \\
\hline $\begin{array}{l}\text { Satisfacción con el } \\
\text { funcionamiento productivo }\end{array}$ & 45 & 27.3 & 87 & 52.7 & 33 & 20.0 \\
\hline Satisfacción social & 44 & 26.7 & 93 & 56.4 & 28 & 17.0 \\
\hline $\begin{array}{l}\text { Satisfacción con el } \\
\text { sentido de vida }\end{array}$ & 31 & 18.8 & 96 & 58.2 & 38 & 23.0 \\
\hline
\end{tabular}

\subsubsection{Resultados 5}

El estudio identificó que existe una relación altamente significativa y negativa entre el uso problemático del internet y la calidad de vida (rho=-.371, p<.01), es decir un nivel alto de calidad de vida en los estudiantes está relacionado con la poca presencia de indicadores de adicción a internet. También se aprecia que existe una relación altamente significativa entre el nivel de satisfacción con la integridad personal y la adicción a internet (rho=-.359, p<.01), es decir un nivel alto de satisfacción con la 
integridad personal está relacionado con la poca presencia de indicadores de adicción a internet. De manera similar, los demás componentes de la calidad de vida están relacionados de manera negativa con la adicción a internet (Ver tabla 8).

Tabla 8

Coeficientes de correlación entre uso problemático del internet $y$ calidad de vida

\begin{tabular}{lcc}
\hline & \multicolumn{1}{c}{ Adicción a internet } \\
\cline { 2 - 3 } & Rho & p \\
\hline Calidad de vida global & -.371 & .000 \\
Satisfacción con la integridad & -.359 & .000 \\
personal & & .000 \\
Satisfacción con el & -.280 & \\
funcionamiento productivo & -.159 & .000 \\
Satisfacción social & -.296 & .000 \\
Satisfacción con el sentido de \\
vida
\end{tabular}

\section{Discusión}

El impacto incalculable de la revolución tecnológica que ha traído consigo cambios en muchas áreas del ser humano, se puede percibir de manera particular en los jóvenes universitarios que están en un proceso de aprendizaje y adaptación a un nuevo rol productivo y social. Dicho rol incluye la posibilidad de formar familias. El uso de internet, cuando es problemático, afecta la calidad de vida de la persona y tiene por lo tanto un efecto en la sociedad.

De los 302 universitarios de la muestra 11.8\% de los varones y un 8.3\% de las mujeres tienen un uso problemático del internet, al incluir los participantes que tienen también problemas frecuentes por sus hábitos de uso de internet se alcanza el 58.9\%. Este dato contrasta con lo hallado por Hilt et al. (2015) quienes en su estudio solo hallaron el $12,81 \%$ de universitarios con riesgo moderado a grave de adicción a internet. El índice de penetración del uso de internet en 2 años y la mayor disponibilidad de redes 
inalámbricas libres en los campus universitarios pueden haber incidido en los resultados entre otros factores.

El estudio confirma la relación negativa altamente significativa entre el uso problemático de internet y la calidad de vida (rho= $-.371, \mathrm{p}<.01)$ este resultado indica que al existir mayor uso problemático del internet la calidad de vida se ve afectada negativamente. Estos hallazgos coinciden con el estudio de Mirzazadeh et al., (2016) donde se encontró que había una correlación negativa entre la adicción a internet de los estudiantes y los componentes físico, sicológico y social de la calidad de vida, dicha investigación encontró que ese uso adictivo de internet, propiciaba el deterioro de las funciones académicas, psicológicas y sociales de los estudiantes, por su parte Tekinarslan (2017) concluyó en su trabajo que el bajo control del uso de internet tenía relación negativa con la calidad de vida.

La presente investigación halló que la satisfacción con la integridad personal (la cual incluye el bienestar físico, emocional y el autocuidado) estaba relacionada de manera significativa y negativa con el uso problemático del internet (rho $=-.371, \mathrm{p}<.01)$, en relación con este resultado PuertaCortés y Carbonell (2013) encontraron que el tiempo de sueño nocturno y las actividades domésticas se veían interferidas por el uso de la red, afectando su bienestar. Por otra parte Jiménez y Pantoja (2007) determinaron que en los sujetos con adicción a internet se puede identificar niveles inferiores de autoestima y sentimientos de vacío, frustraciones, y conductas de aislamiento. Para escapar de estos factores se fortalece la dependencia a la red como medio de establecer interacción social y el bienestar que esto significa, aunque este bienestar es pasajero, a su vez propicia una motivación para permanecer conectado. 
Fue posible establecer que el uso problemático del internet, tenía también una relación significativa de tendencia negativa con la satisfacción con el funcionamiento productivo (rho= -.280, p<.01), que incluye el funcionamiento ocupacional en su trabajo o académico, el desempeño interpersonal y el apoyo social y emocional que la persona acepta. Este resultado se puede observar en contraste con Torres, Duart, Gómez, Marín y Segarra (2016) quienes afirman que uso de internet sobre el rendimiento académico es significativo de manera positiva en el sentido de los estudiantes que lo utilizan para interactuar con sus docentes y compañeros y para investigar y que las malas prácticas y la falta de acceso a internet tenía un efecto negativo sobre el rendimiento académico. Por su parte Jiménez y Pantoja, (2007) de acuerdo a sus hallazgos indicaron que las personas adictas a internet presentaban mayor dificultad al enfrentar la vida cotidiana y responder a las demandas de su entorno social con lo cual se deterioraban sus relaciones.

En concordancia con lo anterior la satisfacción social, la cual está evidenciada en apoyo comunitario y acceso a recursos financieros y de educación, tiene también una relación altamente significativa y negativa con el uso problemático del internet (rho= -.159, $\mathrm{p}<.01)$. Este resultado es acorde parcialmente con el estudio de Matalinares et al. (2016) que indicó una relación directa entre la adicción a internet y la soledad, lo cual permite inferir una baja integración de los jóvenes con UPI a su entorno social, sin embargo el mismo estudio no halló una correlación significativa entre la adicción a internet y el apoyo comunitario. Holden (2011) por su parte halló en su estudio que las personas que utilizan la red para fortalecer relaciones sociales preexistentes y su participación comunitaria, se benefician de manera positiva, además 
concluyó que, la falta de acceso a internet fortalece la exclusión socioeconómica.

En relación al uso problemático del internet y la satisfacción con el sentido de vida que involucra la plenitud espiritual, la plenitud personal y la percepción global de la calidad de vida se halló una relación altamente significativa de tendencia negativa (rho $=-.296, \mathrm{p}<.01$ ), este resultado está acorde con los hallazgos de De Sousa (2014) quien determinó que el uso adictivo de internet presentaba una relación negativa con la espiritualidad cristiana, aunque no con su expresión de fe, el uso de internet interfirió con sus prácticas espirituales. Por otra parte Tarazona (2015) no encontró correlación la cual interpretó desde la perspectiva de una baja accesibilidad a internet por parte de los participantes y el factor de la deseabilidad social para no ser rechazado, por su parte Gupta y Sharma, (2016) no encontraron correlación entre el uso excesivo de internet y la calidad de vida, el uso moderado de internet por el contrario puede favorecer la condiciones de vida pero no tanto como factores relacionados con la empleabilidad y enfermedades físicas y mentales crónicas incluidas en el estudio.

\section{Conclusiones}

Es posible concluir que la falta de control en el uso de internet puede acarrear serias dificultades que se ven reflejadas en una menor calidad de vida. A pesar que las nuevas tecnologías avanzan a un ritmo veloz, no lo hacen al mismo tiempo la formación de nuevos y antiguos usuarios, que permitan un uso adecuado del internet, convirtiéndolo en una valiosa herramienta de trabajo, comunicación, educación y entretenimiento positivo. Los resultados hallados podrían evaluarse en muestras mayores.

\section{Recomendaciones}


Revista de Investigación Universitaria | Volumen 6 - Número 2, Julio - Diciembre, 2017 | ISSN 2078-4015 (En línea)

DOI :

$(\mathrm{co})$ EY

Con base en este estudio es posible recomendar la realización de investigaciones adicionales en relación con estas variables en personas de menor edad, que estén en etapas claves para la formación de hábitos de vida. Igualmente llevar a cabo seminarios de educación en 'Inteligencia tecnológica' entre los jóvenes universitarios y adolescentes de secundaria, con el fin que comprendan el beneficio del uso controlado del internet y las implicaciones del uso problemático del mismo. Considerando la población que presenta problemas frecuentes con el uso del internet, y que potencialmente podrían llegar a un uso adictivo del mismo.

Es esencial integrar al quehacer docente la información y contenidos relevantes para el desarrollo de competencias relacionadas con el uso controlado de internet. Se establece una necesidad y oportunidad de compartir a través de seminarios presenciales, publicaciones virtuales y artículos para el público no académico los resultados de la investigación teniendo como objetivo principal a los padres y maestros.

\section{Referencias}

Alavi, S. S., Eslami, M., Maracy, M. R., Najaf, M., Jannatifard, F., y Rezapour, H. (2010). Psychometric properties of Young Internet Addiction Test. Journal of Behavioral Sciences, 4(3), 185-189. Recuperado de http://journals.bmsu.ac.ir/jbs/index.php/jbs/article/vi ew $/ 232$

Alguacil, J. (1998). Calidad de Vida y Praxis Urbana: Nuevas iniciativas de gestión ciudadana en la periferia social de Madrid (Tesis doctoral). Universidad Complutense de Madrid, España. Recuperado de http://habitat.aq.upm.es/cvpu/acvpu.pdf

Ardila, R. (2003). Calidad de vida: Una definición integradora. Revista Latinoamericana de Psicologia, $\begin{array}{lcr}35(2), & 161-164.0 & \text { Recuperado } \\ \text { http: //www.redalyc.org/pdf/805/80535203.pdf }\end{array}$

de 
Barke, A., Nele, N., y Kröner-Herwig, B. (2012). The German Version of the Internet Addiction Test: A Validation Study. Cyberpsychology Behavior and Social Networking, 15(10), 534-542. Recuperado de http://online.liebertpub.com/doi/abs/10.1089/cyber.2011 .0616

Chang, M., Law, M., y Pui, S. (2007). Factor Structure for the Internet Addiction Test: A Confirmatory Approach. International DSI. Recuperado de http://iceb.nccu.edu.tw/proceedings/APDSI/2007/papers/F inal_82.pdf

Chong, N., Saramah, I., Aili, H., Subash, P., y Manveen, S. (2012). Validity of the Malay Version of the Internet Addiction Test: A Study on a Group of Medical Students in Malaysia. Asia-Pacific Journal of Public Health, 20(10), 1-10. Recuperado de http: //repository.um.edu.my/19529/

Cía, A. H. (2014). Las adicciones no relacionadas a sustancias (DSM-5, APA, 2013): un primer paso hacia la inclusión de las Adicciones Conductuales en las clasificaciones categoriales vigentes. Revista de NeuroPsiquiatria, 76(4), 210-217. Recuperado de http://www. upch.edu.pe/vrinve/dugic/revistas/index.php/ RNP/article/view/1169

Dalbudak, E., y Evren, C. (2014). The relationship of Internet addiction severity with Attention Deficit Hyperactivity Disorder symptoms in Turkish University students; impact of personality traits, depression and anxiety. Comprehensive Psychiatry, 55(3), 497-503. Recuperado de http://www.ercandalbudak.com/wpcontent/uploads/2016/12/A28.pdf

De Sousa-Matías, G. E. (2014). Adicción a internet y participación de la espiritualidad cristiana en estudiantes de la Universidad de Montemorelos en el período académico 2010-2011 (Tesis de maestría no publicada). Universidad de Montemorelos, México. Recuperado de http://dspace.biblioteca.um.edu.mx/xmlui/handle/20.500. $11972 / 396$

Espinosa, F. (2014). Aproximación teórica al concepto de calidad de vida. Revista de Antropología Experimental, 23(23), 331-347. Recuperado de http://revista.ujaen.es/huesped/rae/articulos2014/23esp inosal4.pdf

Frangos, C., Y Kiohos, A. (2010). Internet addiction among greek university students: demographic associations with 
Revista de Investigación Universitaria | Volumen 6 - Número 2, Julio - Diciembre, 2017 | ISSN 2078-4015 (En línea)

the phenomenon, using the greek versión of Young's internet addiction test. International Journal of Economic Sciences and Applied Research, 3(1), 49-74. Recuperado de https://papers.ssrn.com/sol3/papers.cfm?abstract_id=159 1822

Gracia, M., Vigo, M., Fernández, M. J., y Marcó, M. (2002). Problemas conductuales relacionados con el uso de Internet: Un estudio exploratorio. Anales de Psicología, 18(2), 273-292. Recuperado de http://www.um.es/analesps/v18/v18_2/06-18_2.pdf

Grimaldo, M. P. (2012). Calidad de vida en estudiantes de secundaria de la ciudad de Lima. Informes Psicológicos, 20(1), 89-102. Recuperado de http://www.unife.edu.pe/pub/revpsicologia/avances $2012 / \mathrm{m}$ ariagrimaldo.pdf

Gupta, S., y Sharma, R. (2016). The impact of internet usage ( average / excessive ) on quality of life among young adults. Indian Journal of Health and Wellbeing, 7(5), $527-530$.

Recuperado

de https://search.proquest.com/openview/ef2d32b05534a17d3c 63bd173d5808c4/1 ?pq-origsite=gscholarycbl=2032134

Hilt, J., Bouvet de Korniejezuk, R., y Collins, E. (2015). Adicción a Internet en estudiantes universitarios mexicanos. Revista de Investigación Universitaria, 4(2), $11-17$. Recuperado de http://revistascientificas.upeu.edu.pe/index.php/riu/ar ticle/view/681/766

Holden, M. (2011). Life with or without the Internet: the domesticated experiences of digital inclusion and exclusion (Tesis no publicada). London School of Economics and Political Science, UK. Recuperado de http://www.lse.ac.uk/mediallse/research/mediaWorkingPap ers/MScDissertationSeries/2011/68.pdf
IMS corporate, y comScore. (2016). -IMS- Mobile in Latam study 2nd edition. Miami. Recuperado de https: / / www. imscorporate.com/news/Estudios- comScore/IMS-Mobile-Study-Septiembre2016.pdf

Jatuff, D., Zapata-Vega, M. ., Montenegro, R., y Mezzich, J. E. (2007). The multicultural quality of life index in Argentina: a validation study. Actas Españolas de Psiquiatria, 35(4), 253-258. Recuperado de http://repositorio.ub.edu.ar/bitstream/handle/123456789 / 4774 / Jatuff.pdf? sequence=1yisAllowed=y

Jiménez, A. L., y Pantoja, V. (2007). Autoestima y relaciones 
Revista de Investigación Universitaria | Volumen 6 - Número 2, Julio - Diciembre, 2017 | ISSN 2078-4015 (En línea)

interpersonales en sujetos adictos a internet. Psicología - Segunda Época, XXVI(1), 77-89. Recuperado de http://pepsic.bvsalud.org/pdf/repsi/v26n1/v26n1a06.pdf

Khazaal, Y., Billieux, J., Thorens, G., Khan, R., Louati, Y., Scarlatti, E., ... Zullino, D. (2008). French Validation of the Internet Addiction Test. CyberPsychology y Behavior, 11(6), 703-706. Recuperado de https://doi.org/10.1089/cpb.2007.0249

Matalinares, M., Díaz, G., Arenas, C., Rivas, L., Raymundo, O., Baca, D., ... Ventura, J. (2016). Soledad, apoyo comunitario percibido y adicción al internet en adolescentes del Perú. Revista Digital EOS Perú, 8(2), 10-13. Recuperado de https://eosperu.net/revista/wpcontent/uploads/2017/08/art-2-rev-8.pdf

Mezzich, J. E., Cohen, N. L., Ruiperez, M. A., Banzato, C. E. M., y Zapata-Vega, M. I. (2011). The Multicultural Quality of Life Index: presentation and validation. Journal of Evaluation in Clinical Practice, 17(2), 357364. Recuperado de https://doi.org/10.1111/j.1365$2753.2010 .01609 . x$

Miniwatts Marketing Group. (2017). Usage and Population Statistics. Retrieved July 10, 2017, from http: //www.internetworldstats.com/

Mirzazadeh, A., Fatehi, F., Monajemi, A., Sadeghi, A., Mojtahedzadeh, R., y Mirzazadeh, A. (2016). Quality of life in medical students with internet addiction. Acta Médica Iranica, 54(10), 663-667. Recuperado de http://acta.tums.ac.ir/index.php/acta/article/view/5503 $/ 4851$

Moreno, B., y Ximénez, C. (1996). Evaluación de la calidad de vida. In Manual de evaluación en psicología clínica y de la salud (pp. 1045-1070). Madrid: Siglo XXI de España Editores, S.A.,. Recuperado de https://doi.org/10.1017/CBO9781107415324.004

Puerta-Cortés, D., y Carbonell, X. (2013). Uso problemático de Internet en una muestra de estudiantes universitarios colombianos. Avances En Psicologia Latinoamericana, 31(3), 620-631. Recuperado de http://www.scielo.org.co/pdf/apl/v31n3/v31n3a12.pdf

Puerta-Cortés, D., Carbonell, X., y Chamarro, A. (2013). Análisis de las propiedades psicométricas de la versión en español del Internet Addiction Test. Trastornos Adictivos, 14(4), 99-104. Recuperado de http://www.elsevier.es/es-revista-trastornos-adictivos182-pdf-S1575097312700521-S300 
Salas Z., C., y Garzón D., M. O. (2013). La noción de calidad de vida y su medición. The Concept of Quality of Life and Measurement. (English), 4(1), 36-46. Recuperado de http://revistas.ces.edu.co/index.php/ces_salud_publica/ article/download/2751/1984

Sen, A. (1999). La salud en el desarrollo. In 52 Asamblea Mundial de la Salud. Ginebra: Organización Mundial de la Salud. Recuperado de http://apps.who.int/gb/archive/pdf_files/WHA52/swd9.pdf

Şenormancıa, Ö., Saraçlıa, Ö., Atasoya, N., Şenormancı, G., Koktürkc, F., y Atika, L. (2014). Relationship of Internet addiction with cognitive style, personality, and depression in university students. Comprehensive Psychiatry, 55, 1385-1390. Recuperado de https://www.academia.edu/attachments/44901010/download file?st=MTUxNTUzNDcxNCwxOTAuMj QwLjQwLjk2LDI3NTA3Mjg3ys= swp-toolbaryct=MTUxNTUzNDcxNywxNTE1NTM0NzE5LDI 3NTA3Mjg3

Tarazona, D. (2015). Nivel de adicción a internet y nivel de participación en la espiritualidad de un grupo selecto de jóvenes Adventistas de la Asociación del Oriente Colombiano (Tesis de maestría no publicada). Universidad de Montemorelos, Mexico. Recuperado de http://dspace.biblioteca.um.edu.mx/xmlui/bitstream/hand le/20.500.11972/859/Tesis Dorlay Tarazona Jaimes.pdf? sequence=1yisAllowed=y

Tekinarslan, E. (2017). Relationship between Problematic Internet Use, Depression and Quality of Life Levels of Turkish University Students. Journal of Education and Training Studies, 5(3), 167. Recuperado de https://doi.org/10.11114/jets.v5i3.2238

Torres, J. C., Duart, J. M., Gómez, H., Marín, I., y Segarra, V. (2016). Internet use and academic success in university students. Comunicar, 24(48), 61-70. Recuperado de https://doi.org/10.3916/C48-2016-06

Van Roij, A., y Prause, N. (2014). A critical review of "Internet addiction" criteria with suggestions for the future. Journal of Behavioral Addictions, 3(4), 203-213. Recuperado http://real.mtak.hu/51050/1/jba.3.2014.4.1.pdf

White, E. G. (2009). La Educacion. Asociación Publicadora Interamericana.

Young, K. S. (1997). Internet Addiction: Symptoms, Evaluation, And Treatment. In L. Vandecreek, S. Knapp, y T. L. Jackson (Eds.), Innovations in Clinical Practice. Volume 17. Sarasota: Professional Resource Press. Recuperado de 
Revista de Investigación Universitaria | Volumen 6 - Número 2, Julio - Diciembre, 2017 ISSN 2078-4015 (En línea) DOI:

(cc) $\mathrm{BY}$

https://pdfs.semanticscholar.org/27f2/4ddc6b37b1242fabe cd6f35bc05d248c2059.pdf 